\title{
Laser assisted modification of poled silver-doped nanocomposite soda-lime glass
}

\author{
Rokas Drevinskas $^{1,}$, Martynas Beresna $^{1}$, Olivier Deparis ${ }^{2}$ and Peter G. Kazansky ${ }^{1}$ \\ ${ }^{1}$ Optoelectronics Research Centre, University of Southampton, SO17 1BJ, United Kingdom \\ ${ }^{2}$ Solid-State Physics Laboratory, University of Namur (FUNDP), 61 rue de Bruxelles, B-5000 Namur, Belgium \\ *rd1c12@orc.soton.ac.uk
}

\begin{abstract}
Thermal poling assisted homogenization of polydisperse Ag nanoparticles embedded in the soda-lime glass is demonstrated. The homogenization leads to the narrowing of the localized surface plasmon resonance. The subsequent irradiation with linearly polarized ultrashort laser pulses induces spectrally defined and four times larger dichroism than in non-poled sample.
\end{abstract}

Metal-doped nanocomposite glasses are of interest for their unique linear and nonlinear optical properties. Simple and low cost fabrication technique of nanocomposite glass is based on ion exchange. In this technique the metal ions are embedded in glass matrix by ion exchange with further annealing in a reducing atmosphere, resulting in randomly distributed metal aggregates with an exponentially decreasing filling factor across the depth [1]. The optical properties of nanoparticles can then be controlled by ultrashort laser pulses with a wavelength close to localized surface plasmon resonance [2]. However, a non-uniform distribution of nanoparticles in the glass matrix complicates any laser assisted modification. In this work we demonstrate the poling assisted homogenization of Ag nanoparticles embedded in soda-lime glass matrix, which improves laser assisted modification of optical properties.

The polydisperse nanocomposite sample prepared from soda-lime float glass by $\mathrm{Ag}^{+}-\mathrm{Na}^{+}$ion exchange method was provided by CODIXX AG. The spherical Ag nanoparticles of 30-40 nm mean diameter were distributed in a thin surface layer of several micrometers thickness with the high filling factor close to the surface. The sample was thermally poled in air inside the oven using pressed-contact electrodes, with the anode facing the Ag-doped area. The laser modification of $\mathrm{Ag}$ nanoparticles was performed using a regeneratively amplified, mode-locked $\mathrm{Yb}: \mathrm{KGW}$ based ultrafast laser system (Pharos, Light Conversion Ltd.). The laser system was operating at $515 \mathrm{~nm}$ (frequency doubled) and $200 \mathrm{kHz}$ repetition rate. The laser beam was focused on the top of sample via a $\times 5(\mathrm{NA}=0.13)$ objective lens. The modification of poled area was carried out using 1 ps pulse duration, linear polarization, $0.5 \mathrm{~mm} / \mathrm{s}$ writing speed with $5 \mu \mathrm{m}$ interline distance, $10000 \mathrm{pulses} / \mathrm{mm}$ density and the varying laser pulse intensity from $2.2 \times 10^{10} \mathrm{~W} / \mathrm{cm}^{2}$ to $4.4 \times 10^{11} \mathrm{~W} / \mathrm{cm}^{2}$, i.e. below the damage threshold of soda-lime glass. For the modification of pristine area we used $700 \mathrm{fs}$ pulses, linear polarization, $5 \mathrm{~mm} / \mathrm{s}$ speed with $5 \mu \mathrm{m}$ interline distance, 40000 pulses $/ \mathrm{mm}$ density and the intensities from $5.0 \times 10^{10} \mathrm{~W} / \mathrm{cm}^{2}$ to $2.3 \times 10^{11} \mathrm{~W} / \mathrm{cm}^{2}$. For the optical characterization, the absorption spectra of pristine and poled areas were taken with UV-VIS-NIR spectrophotometer (Varian, CARY 500) and optical characterization of modified micro-areas was made with UV-VIS-NIR imaging spectrometer (Andor, Shamrock SR-303i) and VIS microspectrometer system (Olympus BX51, CRAIC). The spectral dependence of modified Ag-doped glass for s- and p-polarizations was measured by inserting linear polarizer before the sample. Images were taken with Olympus BX51 system using $\times 60$ objective lens.

(a)

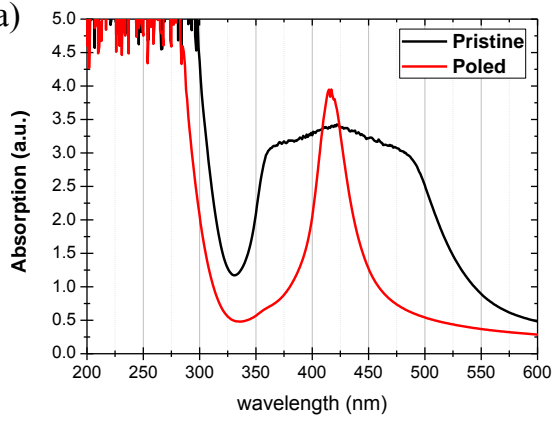

(b)

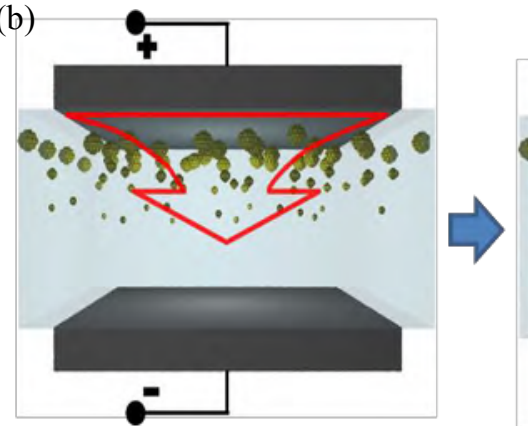

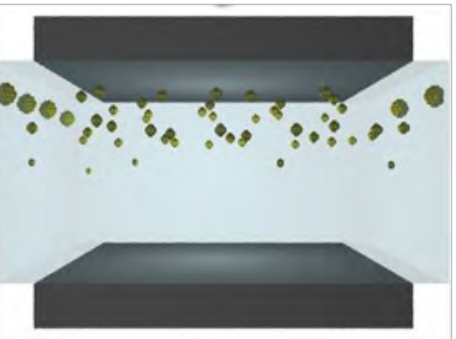

Fig.1. (a) Absorption Spectra of pristine (black) and thermally poled (red) Ag-doped soda-lime glass. (b) Schematic procedure for poling, red arrow indicates the gradient of released $\mathrm{Ag}$ ions.

The thermal poling procedure leads to the ionization of $\mathrm{Ag}$ clusters. The higher the filling factor of $\mathrm{Ag}$ aggregates, the higher the concentration of Ag ions is released [1]. As a result, the top layer is ionized much faster 
than the bottom layers reducing the concentration and size of the nanoparticles located close to the surface. After thermal poling procedure we observe narrowing and increase of the absorption band of Ag nanoparticles (Fig.1) indicating that size distribution of the nanoparticles is reduced. This is a clear evidence of homogenization of Agdoped glass matrix.

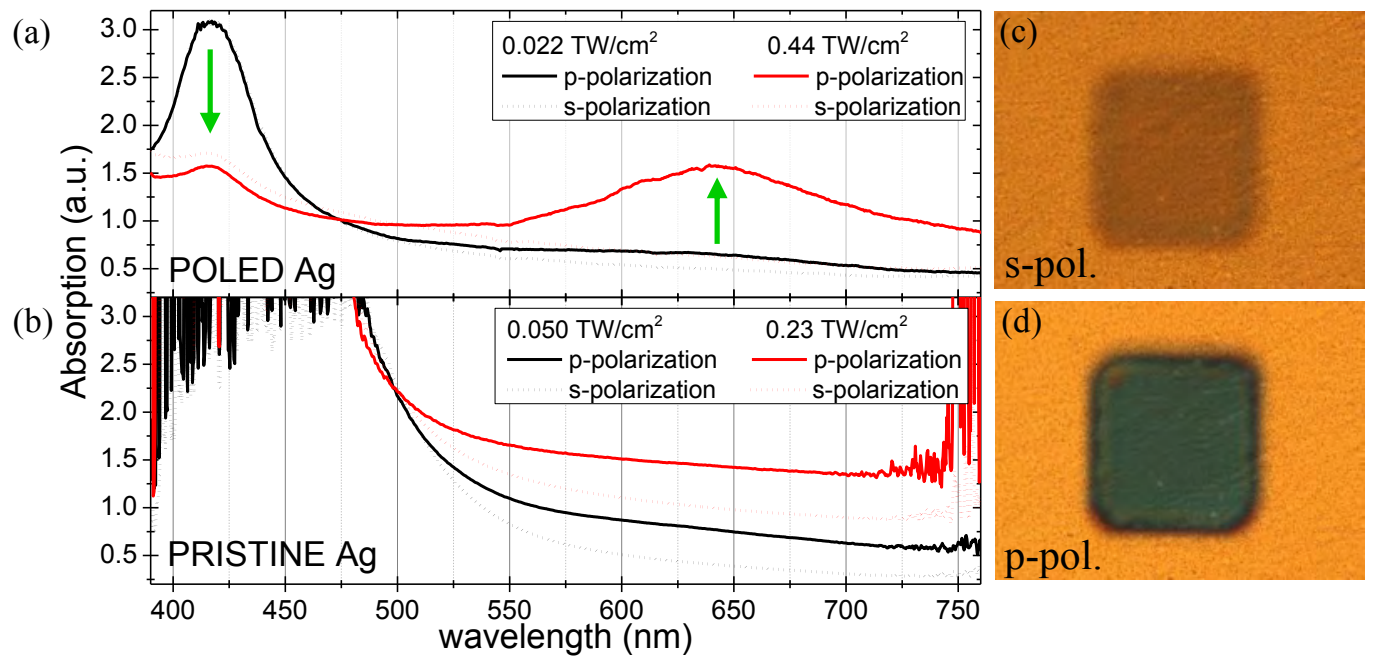

Fig.2. Absorption spectra of (a) poled areas modified with $2.2 \times 10^{10} \mathrm{~W} / \mathrm{cm}^{2}$ and $4.4 \times 10^{11} \mathrm{~W} / \mathrm{cm}^{2}$ laser pulse intensities and (b) pristine areas modified with $5.0 \times 10^{10} \mathrm{~W} / \mathrm{cm}^{2}$ and $2.3 \times 10^{11} \mathrm{~W} / \mathrm{cm}^{2}$, spectra were collected both for s- and p-polarized excitation. (c,d) Images of the poled area modified with the highest energy, for s- and p-polarized illumination, respectively. Green arrows indicate the direction of movement of spectra after the modification.

Ultrashort laser pulse assisted modification of metal-embedded glasses can cause both linear and nonlinear effects due to extremely high peak intensities and the local enhancement of electric field, especially when the irradiated frequency coincides with oscillations of electrons localized on metal surface. As a result Ag clusters in a strong electric field are ionized leading to the shape deformation and dissolution [2, 3]. The deformed Ag nanoparticles exhibit the differential absorption for s- and p-polarizations (Fig. 2). Although the laser pulse frequency is off the peak of SP resonance, we observe strong laser induced dichroism in thermally poled sample. The laser induced dichroism in the poled area was four times larger than in pristine sample at $640 \mathrm{~nm}$. We assume that there is a combination of two states, modified and non-modified Ag clusters, where the interline-unaffected Ag clusters are dominant in the absorption spectra with the peak at $416 \mathrm{~nm}$ and elongated Ag clusters responsible for the SP resonance splitting into two peaks, one around $640 \mathrm{~nm}$ for p-polarized excitation and another very weak below $416 \mathrm{~nm}$ for s-polarized excitation. Combination of thermal poling with ultrafast laser writing can lead to a fine control of optical and structural properties of metal-doped soda-lime glass and can be applied for fabrication of optical components and optical data storage $[4,5]$.

\section{Acknowledgements}

The work was supported by the project FEMTOPRINT, financed by the European Commission Factories of the Future program (FP7/ NMP/Project No 260103), http://www.femtoprint.eu/.

\section{References}

[1] O. Deparis, P. G. Kazansky, A. Podlipensky, et al. "Poling-assisted bleaching of soda-lime float glasses containing silver nanoparticles with a decreasing filling factor across the depth", Journal of Applied Physics 2006, Vol.100(4), pp. Art. No. 044318.

[2] M. Kaempfe, et al. "Ultrashort laser pulse induced deformation of silver nanoparticles in glass," Appl. Phys. Lett. 1999, 74, $1200-1202$.

[3] M. Kaempfe, et al. "Polarization dependence of the permanent deformation of silver nanoparticles in glass by ultrashort laser pulses," Eur. Phys. J. 2001, D 16, 237-240.

[4] A. Royon, et al. "Silver cluster embedded in glass as a perennial high capacity optical recording medium", Advanced materials 2010, 22, 5282-5286.

[5] A. Podlipensky, et al. "Femtosecond laser assisted production of dichroitic 3D structures in composite glass containing Ag nanoparticles," Appl. Phys. 2005, A 80, 1647-1652. 